\title{
Medical Image Classification Using Different Machine Learning Algorithms
}

\author{
Sami H. Ismael \\ sami.hussein@dpu.edu.krd \\ Technical Institute of \\ Bardarash, Duhok Polytechnic \\ University, Duhok, Iraq
}

\author{
Shahab W. Kareem \\ shahab.karim@epu.edu.iq \\ Technical Engineering \\ College, Erbil Polytechnic \\ University, Erbil, Iraq
}

Firas H. Almukhtar

f.almukhtar@cue.edu.krd
Information System Dept., Catholic University, Erbil, Iraq

Accepted on: 02/02/2020

Received on: 28/11/2019

\section{ABSTRACT}

The different types of white blood cells equips us an important data for diagnosing and identifying of many diseases. The automation of this task can save time and avoid errors in the identification process. In this paper, we explore whether using shape features of nucleus is sufficient to classify white blood cells or not. According to this, an automatic system is implemented that is able to identify and analyze White Blood Cells (WBCs) into five categories (Basophil, Eosinophil, Lymphocyte, Monocyte, and Neutrophil). Four steps are required for such a system; the first step represents the segmentation of the cell images and the second step involves the scanning of each segmented image to prepare its dataset. Extracting the shapes and textures from scanned image are performed in the third step. Finally, different machine learning algorithms such as ( $\mathrm{K}^{*}$ classifier, Additive Regression, Bagging, Input Mapped Classifier, or Decision Table) is separately applied to the extracted (shapes and textures) to obtain the results. Each algorithm results are compared to select the best one according to different criteria's.

Keywords: Machine learning (ML), Classification, Segmentation, digital image, image extraction, and histogram

$$
\begin{aligned}
& \text { تصنيف الصور الطبية باستخدام خوارزميات التعلم الآلي المختلفة } \\
& \text { فراس هشام المختار } \\
& \text { شهاب وهاب كريم }
\end{aligned}
$$

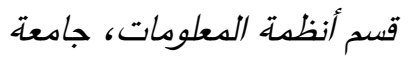

$$
\begin{aligned}
& \text { كلية الهندسة التقنية، جامعة } \\
& \text { بوليتكنيك، أربيك، العراق القيه، جامبه }
\end{aligned}
$$

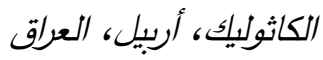

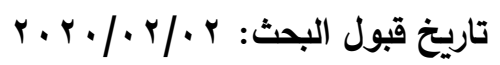

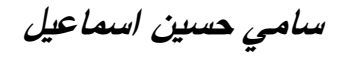

$$
\begin{aligned}
& \text { الدعهد التقني بردش، جامين اسعاعيل }
\end{aligned}
$$

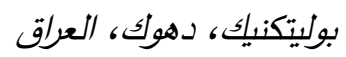

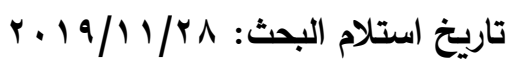

\section{|لملغص}




$$
\begin{aligned}
& \text { يطبق بشكل منفصل على المستخرج (الأشكال والنسيج) للحصول على النتائج. تتم مقارنة نتائج كل خوارزمية } \\
& \text { لتحديد أفضلها وفقًا لمعايير مختلفة. } \\
& \text { الكلمات المفتاحية: التعلم الآلي (ML)، التصنيف، التجزئة، الصور الرقمية، استخراج الصور، الرسم البياني. }
\end{aligned}
$$

\section{Introduction}

In medicine, further especially those areas of hematology and dangerous infections, classifying various types of blood cells can be utilized as tools in the medical analysis, by counting the repetitions of individual cells and match it with the number taken from the normal blood samples. The examination of the microscopic images of blood cells shows an important knowledge about the patient's health. Nevertheless, the examination of blood cells through hand-operated mechanism is sensitive to wrong, slow process and an extremely time-consuming by means of the various morphological highlights concerning the cells [1].

Jaroonrut and Charnchai [2] proposed a method that involves a classification, feature extraction, nucleus segmentation, pre-processing, feature selection, and cell segmentation to decide about identifying the blood ailments.

Using Otsu's threshold techniques, Anjali and Bhadauria [3] try to limit the nucleus from blood smear images then work on expanding the histogram of the image after the application of special filters to decrease image noise and rising the illumination of nucleus. A mathematical morphological process are used to extract some elements not considered as WBCs, then shape-based characteristics are selected to classify them into different categories.

Sedat and Karhan [4] proposed an automatic method for counting, doing size measurement, and then classifying the white blood cells according to genetics methods. Siddhartha and Bibek [5] propose a computerized system that can use several image processing techniques to recognize the white blood cell found in a microscopic image of a human blood samplewith the aid of Neural Network methods. In this paper, a dataset with hundreds of images of white blood cells are examined and classified depending on several methods. The classification process will help to diagnose particularly each disease in a quick and automatic way. Pathological specialist's works on several examinations of blood to analyze and diagnose. Some of these analyses are concentrating on the elements of blood itself; others work on the elements detected in the blood to recognize irregular functioning from different members.

Therefore, we introduce a method that will support pathologists for recognizing quantity of blood cell depending on the process of segmentation which lead to support the detection of the diseases. In our work, a special enhanced algorithm is used to extract and segment the WBC, then, machine learning algorithms are utilized for the classification process to get the ultimate results.

The sections of this paper can be organized as follows: section one is the introduction, while the second section explains the Image Pre-processing. The third section displays the machine learning algorithms that are used for classifications, and our proposed method illustrates in the fourth part. Analysis and results will be explained in the fifth part. The final section will clarify the conclusions of this study followed by references.

\section{Image Preprocessing}

Through the various applications of real-time medical diagnostics, digital image processing becomes very necessary in the field of health regard. Some of the data are 
imperfect and not clear, also these data are expected to include unnecessary and repetitive information or errors [6]. Data preprocessing, which is an essential part of data digging techniques, used to help reconstructing the raw data to an acceptable format [7]. Then machine learning techniques are performed to do a significant task in the process of data analyses. This section outlines some important data managing techniques, including data purifying, data transformation, and data modulation. Data preprocessing in general, consists of converting the collection of data attributes to another form in order to allow the appropriate data mining or machine learning technique to obtain better results [8]-[11]. Classifier development is one of the commonly researched issues within data mining and machine learning areas, comes with thousands of algorithms and models. The quality of these learned models depends on the essence of the training data. There is no way to discover which classifier inducer is good to apply. Especially, if the training data are unreliable, then bad models will result [12]. In common, digital image processing involves four major fields (Figure. 1) as shown below.

Image formation holds all the levels of obtaining the image form a digital matrix. Image visualization process uses all kind of manipulations to the image matrix so as to produce an image optimized product. In the analysis process of the image, all levels of processing, which is applied on quantitative measurements are used for detailing the biomedical pictures.

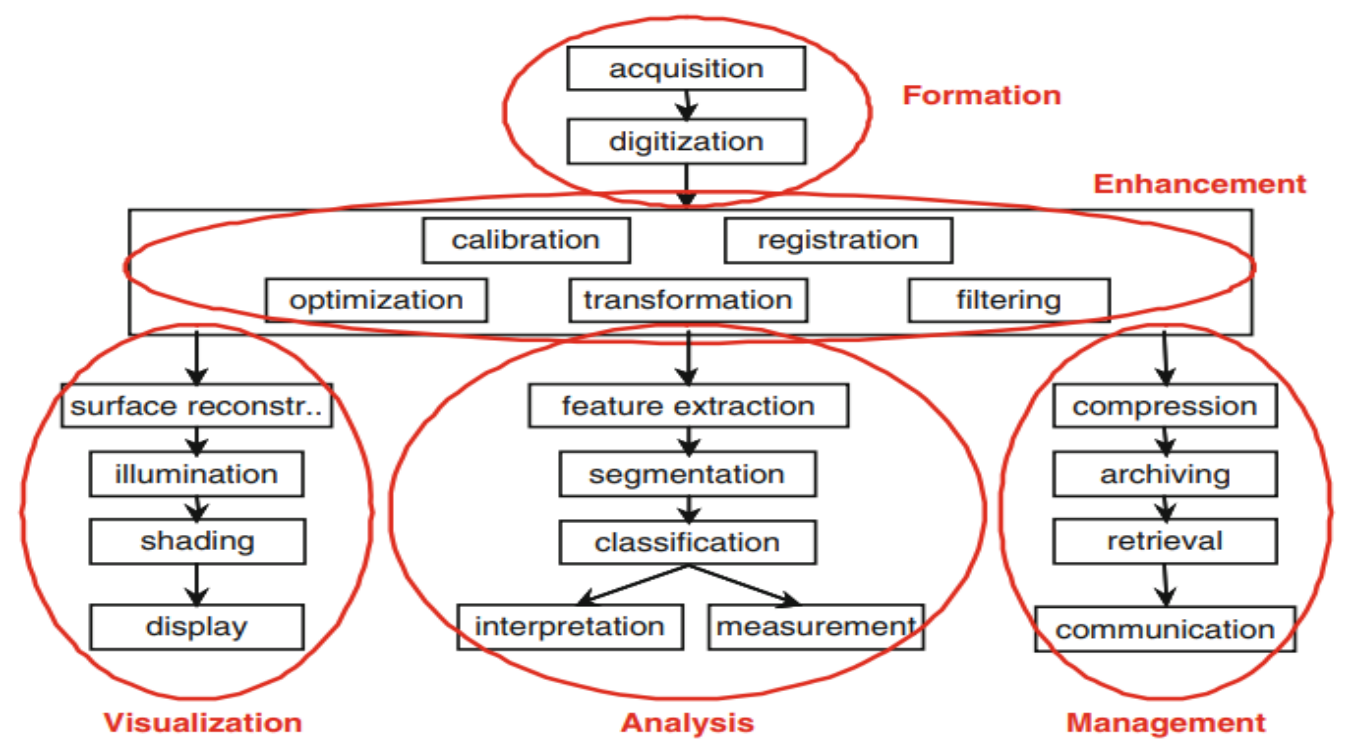

Figure 1: Image Processing Module [12].

All of these operations need a priori information on the kernel of the image details, which must be merged with algorithms to work on the highest level of abstraction. Subsequently, the image analysis method is very definite, and enhanced algorithms works in a quick way into other usage areas. Image administration gathers all the methods that includes efficient warehouse, communication, archiving, transmission, and image data retrieval (access). So, the methods of telemedicine are also considered as a section of the image administration [13]. Indifference to image analysis, usually pointed to as a high-level image processing, low-level processing means standard or 
impulsive procedures that can be achieved with no need for any information's about the images. This kind of algorithms has related wares despite the content of the images [13]. The primary step in this method needs a tracer for image to transform the signal into digital image, or images can be grabbed in RGB color format from online medical library or clinic blood sample images and then changed into a grayscale level. Figure 2a shows a human blood cell with four WBCs and also several RBCs (Red Blood Cells) all under microscopic perspective [6].

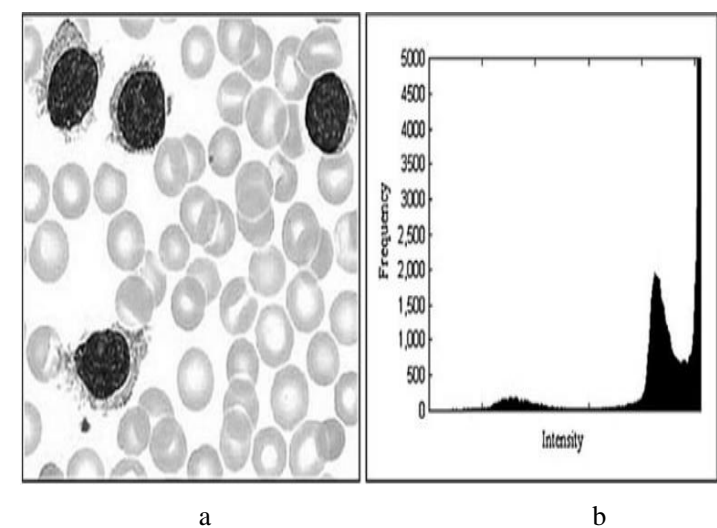

Figure 2: (a) Microscopic image of human blood cell (b) histogram of the image

In blood cell image exposure, enhancement methods are very important, for the objective of reducing noise. Cell segmentation requires the elimination of background containing RBC's, platelets, and different things of the image obtained. White blood cells, the objects of powers, grow as the product of the segmentation process. Accurate segmentation should result in a complete white blood cell, including the nucleus and the cytoplasm. The form of the nucleus, its area, texture, and the proportion of its content in the cell are considered to be some of the features that are needed to classify the cell.

Image segmentation is the most significant step in image processing, and it will instantly change succeeding processing. By scientific theories, image segmentation has performed excellent development and a lot of novel segmenting algorithms have been introduced. However the best methods have their own defects. As for cell images, owing to the complicated universe, it nevertheless continues a challenging responsibility to separate and counts them [7].

\section{Classification Algorithms}

The operations of ML can be utilized after scanning the image, converting it to binary and then segmented for further processing. A vertical and horizontal scanning of the image is needed to set the values of (top, right, left and bottom) borders needed to surround the region of the investigated cell [14]. Machine learning (ML) is an algorithm set particularly agreed to forecast. Those ML techniques are simple to perform also offer better results than the standard mathematical approaches to classify the images [15].

Classification techniques can predict a confident result depending on the offered input. To predict the result, the technique uses a collection of properties for the purpose of training and the result, will be announced as either object or forecast characteristic. The classification technique selects pixels in the image to sections or categories of concern. There are couple models of classification algorithms supervised, and 
unsupervised. Supervised classification utilizes the phantom marks received from training samples unless data to analyze dataset or image. Unsupervised classification identifies spectral classes in a multiband image without the analyst's intervention. The unsupervised Image Classification algorithms uses a technology to build a set of clusters then investigate the quality of the clusters which in turn passes the result to the decision algorithm [16].

To classify the set of data into various subsets or classes, the correlation between the classes and the data within which they are classified are necessarily be known. In this paper, five machine learning algorithms will be presented, the mathematical model behind these algorithms is illustrated as follow:

\section{A. K-Star Classification Algorithm:}

$\mathrm{K}$-star or $\mathrm{K}^{*}$ is a classifier that work on instance-based technique. This algorithm try to examine the instance if it is related to any of the training dataset using a correlation function. This method deviates from different instance-based learners in that it uses a function called entropy-based function. This function classifies the situation by assigning it to the set of data that are pre-defined and classified models. The important thing about this theory is that comparable situations bestowal own similar classifications [17].

\section{B. Additive Regression:}

Meta classifier that improves the appearance of a regression base classifier. All repetition provides a pattern to the residuals devised by the classifier on the prior iteration. The forecast is achieved by attaching the forecasts of any good classifier. Overcoming the reduction (learning rate) parameter supports limit overfitting and produces a smoothing impact but enhances the learning experience [18].

\section{Bagging:}

Bagging is a technique for enhancing the outcomes of machine learning classification algorithms. This technique was expressed by Leo Breiman and its title was understood of the expression "bootstrap aggregating" [19]. In the state of classification within couple possible groups, a classification algorithm produces a classifier H: D $Æ\{-1,1\}$ on the principle of a foundation set of example information D. The bagging technique builds a series of classifiers $\mathrm{Hm}, \mathrm{m}=1, \ldots, \mathrm{M}$ in regard to qualifications of the training set. These classifiers are mixed into a composite classifier [20].

\section{Input Mapped Classifier:}

Envelope classifier that labels inappropriate training and test data by developing a mapping within the training data that a classifier has produced including the incoming test situations' construction. Model characteristics that are not observed in the incoming situations obtain missing values, then take incoming unnecessary characteristic values that the classifier has not observed previously. A new classifier can be trained or an actual one stored from a file [21].

\section{E. Decision Table:}

A Decision Table is a technique utilized to produce a full set of test cases without utilizing the inherent construction of the application in issue. In sequence to produce test instances, we utilize a table to receive the input and output values of a program. Such a table is divided up into four parts (Stub portions, Actions, Entry portions, and Conditions) [22]. 


\section{Algorithms and Experiments}

\section{A. Methodology}

Image enhancement is the starting step in the pre-processing steps which involves performing a set of operation required for better further processing. These include histogram equalization to enhance the contrast (we will call the image in this step as X) then apply linear contrast stretching (result will be called Y) to whiten the image in general. Later on, highlight the entire image object and take off all the ingredients of the blood with the minimum effect of malformation. Another step is to decrease the image din and apply a global threshold to convert it to a binary image. Finally, apply morphological process for removing small pixel groups. In the operation of morphology, the state of the pixels of the output image depends on the correlation of the input image with its neighbor similar pixels. Working on this image depend on the shape, the size of the structure component and on the amount of the pixels removed or added.

Various morphological processes utilize the method of Dilation and Erosion in dealing with objects inside the image. Dilation a technique that add pixels to the borders of the objects in an image, while erosion technique is used to delete some of the unnecessary pixels from the object borders. The suggested system tries to scan the image and use these two techniques to extract the white shapes inside the image and neglect the black one's adding or removing some pixels from the shapes borders as necessary.

The proposed system tries to scan the image vertically from top to bottom, registering the highest pixel values from each cell region into a specific list, then repeat the same scanning process horizontally. Now, the final list contains all the information's needed about the objects exists in the image. This method increases processing efficiency and make it easy for the next step to be performed.

Feature selection mechanisms are extremely influenced by the performance of the classifier chosen and vice versa. To get robust and powerful classifications, the features must be enough to describe all WBC subgroup in a perfect way, and also keep these WBC isolated from each other. Our proposed system creates an N-dimensional table of feature vectors which includes texture, shape and intensity features. After extracting the significant features from each $\mathrm{WBC}$, vectors of the data are named according to that feature. A subsequent action is taken by converting the feature vectors into a collection of attributes used by the classifier procedures. Different classification techniques are used to deal the WBC identification process.

\section{B. Experimental Results}

The WBC microscopic images that are used for the experiments have been taken from the central public health Laboratory in Duhok city - Kurdistan Region, Iraq. Images are captured from smear slides by a Nikon 50i microscope, equipped with a Nikon color camera DP5M.

Before presenting and discussing the result, we start to present the criteria (Correlation coefficient, Mean absolute error, Root-mean-square deviation, Relative absolute error, and Root relative squared error) used to produce the output. The first criteria are the correlation coefficient which measures the firmness and the trend of a linear correlation between two variables. The values of this criteria extent from 0 to 1 . The nearer the absolute value to 1 , is the solid the relationship is. Zero means weak relationship between the variables [23]. 
Mean Absolute Error (MAE) is a statistical fault measurement so that this method calculates the average of the distances between each two values of the real data $\left(Z_{t}\right)$ and fitted predicted data $\left(Z^{\prime}{ }_{t}\right)$ points. MAE is calculated by taking the average of the absolute errors and is most appropriate when the cost of forecast errors is proportional to the absolute size of the forecast errors. MAE is given by:

$$
\left.\mathrm{MAE}=\frac{1}{N} \sum_{t}^{N}\left|Z^{\prime}{ }_{t}-Z_{t}\right|\right)=\frac{1}{N} \sum_{t}^{N}\left|e_{t}\right|
$$

Suppose, $\left(\mathrm{e}_{1, \mathrm{t}}, \mathrm{e}_{2, \mathrm{t}}\right), \mathrm{t}=1,2, \ldots \mathrm{m}$ are $\mathrm{h}$-step out-of-sample forecast errors of models 1 and 2, respectively. Taking MAE as a measure of prediction loss, the loss differential from the two models can be expressed as $d t=\left|e_{1 t}\right|-\left|e_{2 t}\right|, t=1,2, \ldots m$ [24]. The Root Means Square Deviation (RMSD) (has another name (Root Mean Square Error (RMSE)) is used to find the diveations between values predicted by a model and the values actually spotted from the real model. These solo deviations are named residuals, the RMSD works to sum them all into a single measure of predictive power. The RMSD of the prediction model with respect to the estimated variable $\mathrm{X}_{\text {model }}$ is defined as the square root of the mean squared error:

$$
R M S D=\sqrt{\frac{\sum_{i=1}^{n}\left(X_{o b s, i}-X_{\text {model }, i}\right)^{2}}{n}}
$$

Such that $X_{\text {obs }}$ is observed values and $X_{\text {model }}$ is modeled values at time/place $i$. The RMSD values can be used to differentiate between the model performances in a calibration period, with a validation period to compare the individual model performance to the predictive models. RMSD can be found by taking square root of the average of each squared errors. So, the effect of each error on RMSD is so dependable on the size of the squared error; larger errors have a disproportionately large effect on RMSD [24].

Relative Absolute Error (RSE) is the difference degrees between the absolute deviation obtained from the prediction model and the one obtained by directly speculating the training sample. It is inversely proportional to prediction accuracy. The minimum the RSE is, the maximum the accuracy of the prediction can be:

$$
R S E=\frac{\sum_{i=1}^{n}\left|f_{i}-y_{i}\right|}{\sum_{i=1}^{n}\left|f^{\prime}{ }^{\prime}-y_{i}\right|^{\prime}}
$$
follows:

RRSE (Root Relative Squared Error) is also used and can be calculated as

$$
R R S E=\frac{\sum_{i=1}^{n}\left|f_{i}-y_{i}\right|^{2}}{\sum_{i=1}^{n}\left|f^{\prime}{ }_{i}-y_{1}\right|^{\prime 2}}
$$

RRSE which is also inversely proportional to prediction accuracy. The minimum the RRSE is, the maximum the prediction accuracy can be [25]-[26].

A group of microscopic medical pictures were captured from the central public health Laboratory in Duhok city for investigation. After applying the mentioned classification algorithms in section III, we present the results of different criteria as shown in Table 1. The output of the classification algorithms are divided into five different models (Basophil, Eosinophil, Lymphocyte, Monocyte, and Neutrophil). 


\begin{tabular}{|c|c|c|c|c|c|}
\hline \multicolumn{7}{|c|}{ Table 1: Evaluation of WBC images classification } \\
\hline & $\begin{array}{c}\mathrm{K}^{*} \\
\text { classifier }\end{array}$ & $\begin{array}{c}\text { Additive } \\
\text { Regression }\end{array}$ & Bagging & $\begin{array}{c}\text { Input } \\
\text { Mapped } \\
\text { Classifier }\end{array}$ & $\begin{array}{c}\text { Decision } \\
\text { Table }\end{array}$ \\
\hline $\begin{array}{c}\text { Correlation } \\
\text { coefficient }\end{array}$ & 1 & 0.9316 & 0.7974 & 0 & 0.9874 \\
\hline $\begin{array}{c}\text { Mean } \\
\text { absolute } \\
\text { error }\end{array}$ & 0 & 0.4578 & 0.8218 & 1.5733 & 0.06 \\
\hline $\begin{array}{c}\text { Root mean } \\
\text { squared } \\
\text { error }\end{array}$ & 0 & 0.5466 & 0.9526 & 1.6813 & 0.2236 \\
\hline $\begin{array}{c}\text { Relative } \\
\text { absolute } \\
\text { error }\end{array}$ & 0 & $38.15 \%$ & $68.49 \%$ & $100 \%$ & $5 \%$ \\
\hline $\begin{array}{c}\text { Root } \\
\text { relative } \\
\text { squared } \\
\text { error }\end{array}$ & 0 & $38.65 \%$ & $67.36 \%$ & $100 \%$ & $15.81 \%$ \\
\hline
\end{tabular}

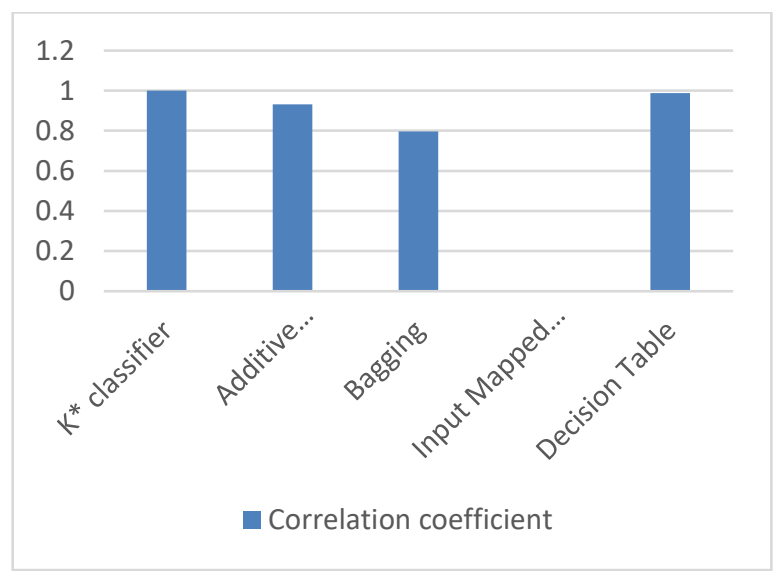

Figure 3: Correlation Coefficient

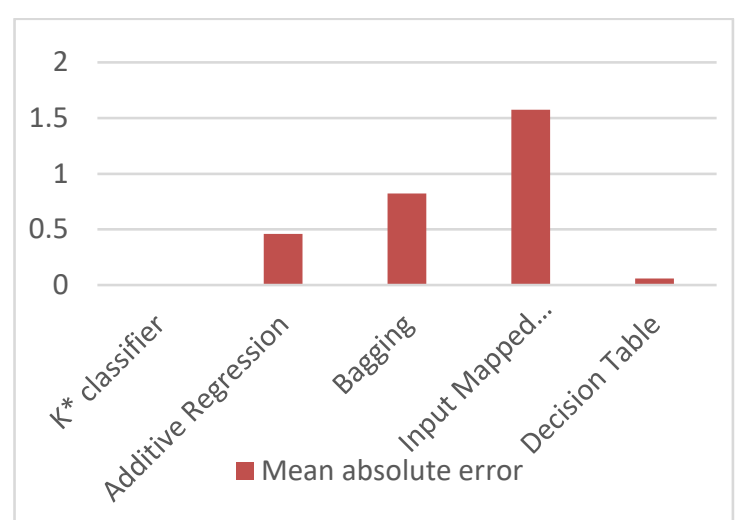

Figure 4: Mean absolute error 


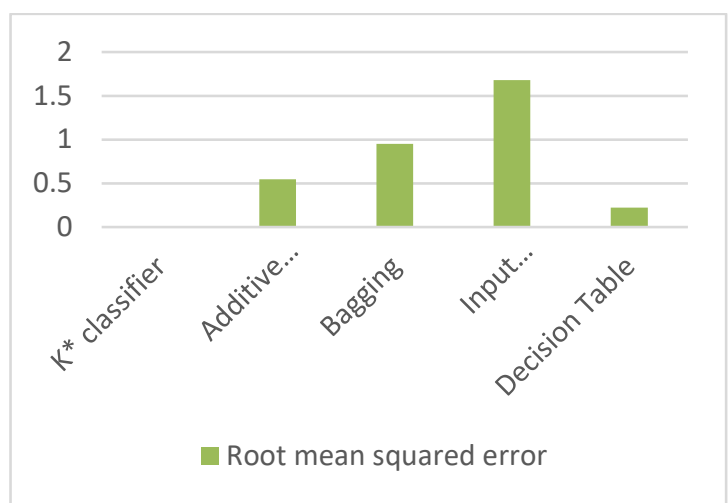

Figure 5: Root mean squared error

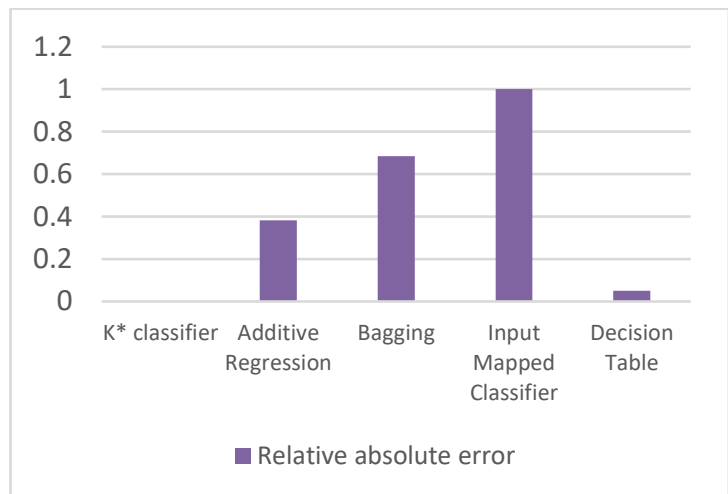

Figure 6: Relative Absolute Error

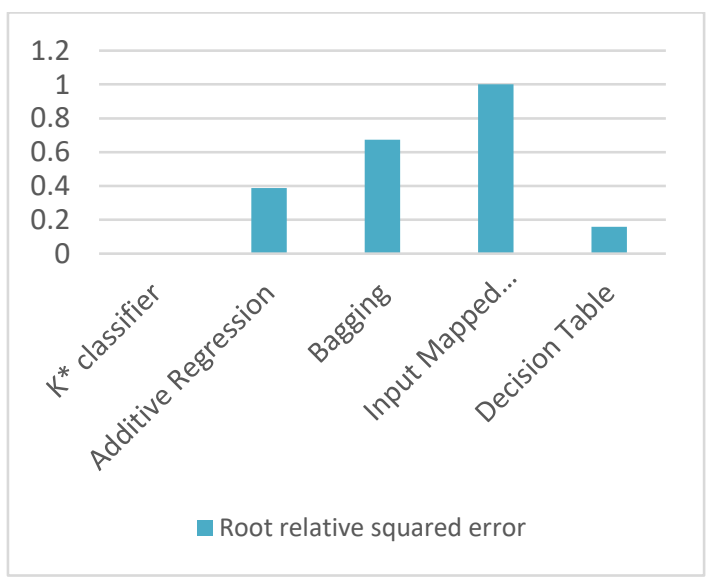

Figure 7: Root relative squared error

From the figures above (Fig 3 - Fig 7), we can conclude that the $\mathrm{K}^{*}$ classifier is better than the other mention algorithms followed be Decision table algorithm.

\section{Conclusion}

In this paper, we proposed a system that automatically identifies the existence of the WBCs in the medical images. It also does image segmentation, improvement and classification. Different types of measurements are performed for the classification step such as (Root-mean-square deviation, Correlation coefficient, Relative absolute error, Mean Absolute Error, Root relative squared error and $\mathrm{K}^{*}$ algorithms). According to the results of these measurements, the WBC images are classified into five types (Basophil, Eosinophil, Lymphocyte, Monocyte, and Neutrophil). 
The experimental results show that $\mathrm{K}^{*}$ classifier is the best among all the others to work on our dataset. The operations of morphological analysis are used to decrease the image corrodes and to improve the important features (Image intensity, shape, texture, and color) during the identification process. In future works, we will extend this study to work on another method of medical image classification. 


\section{$\underline{\text { REFERENCES }}$}

[1] MINAL, D. J., Atul H. K. and SURALKAR, R. S. (2013) White Blood Cells Segmentation and Classification to Detect Acute Leukemia. Vol. 2, Issue 3. Maharashtra: International Journal of Emerging Trends \& Technology in Computer Science.

[2] Jaroonrut Prinyakupt and Charnchai Pluempitiwiriyawej,"Segmentation of white blood cells and comparison of cell morphology by linear and naïve Bayes classifiers", BioMed Eng OnLine (2015).

[3] Anjali Gautam; Harvindra Bhadauria, "Classification of white blood cells based on morphological features", International Conference on Advances in Computing, Communications and Informatics (ICACCI), IEEE, 2014.

[4] Sedat Nazlibilek, Deniz Karacor, Korhan Levent Ertürk, Gokhan Sengul, Tuncay Ercan, Fuad Aliew,"White Blood Cells Classifications by SURF Image Matching, PCA and Dendrogram",

[5] Siddhartha Banerjee, Bibek Ranjan Ghosh, Surajit Giri and Dipayan Ghosh, "Automated System for Detection of White Blood Cells in Human Blood Sample”, Springer Nature Singapore Pte Ltd. 2018.

[6] Samir K. Bandyopadhyay,"METHOD FOR BLOOD CELL SEGMENTATION", Journal of Global Research in Computer Science, Volume 2, No. 4, April 2011.

[7] Kuhn, Max, and Kjell Johnson. Applied predictive modeling. New York: Springer, 2013.

[8] Amin Salih Mohammed, Shahab Wahhab Kareem, Ahmed khazal al azzawi,Dr.M. Sivaram. (2018). Time Series Prediction Using SRE- NAR and SRE- ADALINE. Jour of Adv Research in Dynamical \& Control Systems, 17161726

[9] Quinlan J. R.: C4.5: Programs for Machine Learning. Morgan Kaufmann, 1993.

[10] Tan P.-N., Steinbach M., Kumar V.: Introduction to Data Mining. Addison Wesley, Boston, MA, 2006.

[11] Zeebaree, D. Q., Haron, H., Abdulazeez, A. M., \& Zebari, D. A. (2019, April). Trainable Model Based on New Uniform LBP Feature to Identify the Risk of the Breast Cancer. In 2019 International Conference on Advanced Science and Engineering (ICOASE) (pp. 106-111). IEEE.

[12] Faisal Kamiran - Toon Calders, "Data preprocessing techniques for classification without discrimination", Knowl. Inf. Syst. 2012) 33:1-33 DOI 10.1007/s10115-011-0463-8.

[13] Thomas M. Deserno, "Biomedical Image Processing”, Springer-Verlag Berlin Heidelberg 2011.

[14] HUIYU Z., JIAHUA,W. and JIANGUO, Z. "Digital Image Processing Part II." ,bookboon, London, 2014. 
[15] Sehla Loussaief, Afef Abdelkrim, "Machine Learning Framework for Image Classification", 7th International Conference on Sciences of Electronics, Technologies of Information and Telecommunications (SETIT), 2016.

[16] Tanmoy Das, "Machine Learning algorithms for Image Classification of hand digits and face recognition dataset", International Research Journal of Engineering and Technology (IRJET) e-ISSN: 2395-0056 Volume: 04 Issue: 12 | Dec-2017.

[17] Deeman Y. Mahmood, Dr. Mohammed A. Hussein, "Intrusion Detection System Based on K-Star Classifier and Feature Set Reduction", IOSR Journal of Computer Engineering (IOSR-JCE) Volume 15, Issue 5 (Nov. - Dec. 2013).

[18] J.H. Friedman, "Stochastic Gradient Boosting”, Stanford University, 1999.

[19] Breiman, L.: Bagging predictors. Technical Report 421, Department of Statistics, University of California at Berkeley, 1994.

[20] Kristína Machová, František Barčák, Peter Bednár, ”A Bagging Method using Decision Trees in the Role of Base Classifiers", Acta Polytechnica Hungarica Vol. 3, No. 2, 2006.

[21] Remco R. Bouckaert, Eibe Frank, Mark Hall, Richard Kirkby, Peter Reutemann, Alex Seewald, David Scuse, "WEKA Manual for Version 3-7-8", University of Waikato, Hamilton, New Zealand, 2013.

[22] Dr. Roggenbach and Prof. Schlingloff, "A Review Paper on Decision TableBased Testing", Cai Ferriday 345399, January 7th, 2007.

[23] A. G. Asuero, A. Sayago, and A. G. Gonz 'alez, "The Correlation Coefficient: An Overview", Critical Reviews in Analytical Chemistry, Taylor and Francis Group, LLC,36:41-59, 2006.

[24] Naveen Kumar Boiroju, Ramu Yerukala, M. Venugopala Rao and M. Krishna Reddy, "A BOOTSTRAP TEST FOR EQUALITY OF MEAN ABSOLUTE ERRORS", ARPN Journal of Engineering and Applied Sciences, VOL. 6, NO. 5, MAY 2011.

[25] Joshua M. Kantor, Christ D. Richmond, Daniel W. Bliss, and Bill Correll, "Mean-Squared-Error Prediction for Bayesian Direction-of-Arrival Estimation", IEEE TRANSACTIONS ON SIGNAL PROCESSING, VOL. 61, NO. 19, OCTOBER 1, 2013.

[26] T. E. Hoff, R. Perez, J. Kleissl, D. Renne, and J. Stein, "Reporting of irradiance modeling relative prediction errors," Progress in Photovoltaics: Research and Applications, vol. 21, no. 7, pp. 1514-1519, 2013. 


\section{Appendix}

\begin{tabular}{|c|c|c|c|c|c|c|c|c|c|}
\hline $\begin{array}{l}\text { à } \\
\text { 인 } \\
\text { L }\end{array}$ & 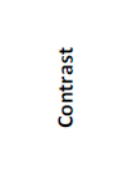 & 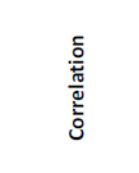 & 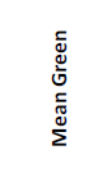 & & $\frac{\stackrel{0}{\frac{J}{*}}}{\frac{\underline{\underline{z}}}{\mathrm{t}}}$ & $\begin{array}{l}\frac{n}{u} \\
\underline{\underline{t}} \\
\frac{t}{3}\end{array}$ & 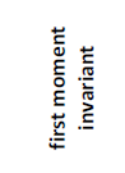 & 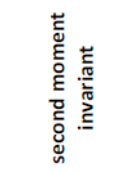 & 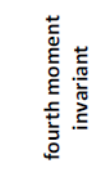 \\
\hline 0.200622 & 2.409167 & 0.791370342 & 86.52678 & 80.36623327 & 86.54224658 & 0.0155068 & 1.3501619 & 0.0003048 & -0.932115 \\
\hline 0.200622 & 1.54788 & 0.799252098 & 39.2102 & 65.42869607 & 85.19787762 & 0.0423920 & 0.5868243 & -1.8167282 & -3.134586 \\
\hline 0.498028 & 1.979021 & 0.769271031 & 43.32055 & 70.27339895 & 75.64327523 & 0.0253650 & 0.6816674 & -2.4648080 & -1.226830 \\
\hline 0.696212 & 0.502877 & 0.92567521 & 63.83442 & 56.15236267 & 102.6867384 & 0.0532020 & 0.3631059 & -6.1140653 & -1.990842 \\
\hline 0.696212 & 0.438805 & 0.936331884 & 59.69666 & 55.20995029 & 108.5470018 & 0.0494209 & 0.6006997 & -3.5552895 & -2.988857 \\
\hline 0.696212 & 0.372064 & 0.908282978 & 90.23743 & 46.18562384 & 81.93405223 & 0.0701230 & 0.2091661 & -8.2779717 & -6.301911 \\
\hline 0.696212 & 0.335548 & 0.907278182 & 81.89769 & 45.6507388 & 78.23567242 & 0.0635273 & 0.3109673 & -2.1897931 & -5.069425 \\
\hline 0.696212 & 0.292147 & 0.954639826 & 67.87741 & 55.55648574 & 104.1520814 & 0.0580691 & 0.3979307 & -3.7084227 & -1.395470 \\
\hline 0.696212 & 0.278464 & 0.958972145 & 67.15405 & 55.16663243 & 103.1766932 & 0.1602457 & 0.1581838 & -3.9921327 & -3.316172 \\
\hline 0.696212 & 0.403647 & 0.920532717 & 71.2965 & 48.78551717 & 94.91355894 & 0.0501564 & 0.5219770 & -3.1909033 & -4.506538 \\
\hline 0.696212 & 0.500186 & 0.908546685 & 62.08148 & 50.26094996 & 99.41495807 & 0.0506595 & 0.2927502 & -2.7257209 & -3.324992 \\
\hline 0.757878 & 0.303377 & 0.945792927 & 58.17798 & 49.92372501 & 100.6597611 & 0.0727058 & 0.2268232 & -4.8344255 & -3.601006 \\
\hline 0.757878 & 0.325235 & 0.909523183 & 75.50045 & 41.39269398 & 86.31761522 & 0.1337262 & 0.2736336 & -3.9795358 & -4.641022 \\
\hline 0.757878 & 0.449557 & 0.887898524 & 85.01325 & 8.21961968 & 77.92691873 & 0.0810591 & 0.0739594 & -4.77888875 & -7.664978 \\
\hline 0.757878 & 0.313162 & 0.908730621 & 73.33601 & 41.67185844 & 83.87253962 & 0.0453283 & 0.2536981 & -3.3726692 & -3.681453 \\
\hline 0.757878 & 0.449557 & 0.887898524 & 85.01325 & 48.21961968 & 77.92691873 & 0.0810591 & 0.0739594 & -4.7788875 & -7.664978 \\
\hline 0.757878 & 0.617458 & 0.855609141 & 92.74314 & 46.51432153 & 78.94638215 & 0.1014697 & 0.1128292 & -7.3067151 & -6.418477 \\
\hline 0.757878 & 0.584271 & 0.905460964 & 81.44395 & 56.2321388 & 95.22334903 & 0.0448654 & 0.1644957 & -6.1824146 & -5.463053 \\
\hline 0.757878 & 0.545774 & 0.910168255 & 81.35618 & 55.668998811 & 94.99144143 & 0.0518240 & 0.2405178 & -8.6666225 & -6.592553 \\
\hline 0.78556 & 0.529396 & 0.86516031 & 73.83992 & 46.02854593 & 79.20876759 & 0.0396892 & 0.0415815 & -3.3115209 & -5.692704 \\
\hline 0.78556 & 0.33483 & 0.923594539 & 87.55686 & 45.7234666 & 82.45292483 & 0.1273536 & 0.4893525 & -2.6328469 & -8.009744 \\
\hline 0.811278 & 0.411526 & 0.895056652 & 93.03763 & 42.1665069 & 78.8459894 & 0.1615340 & 0.2950990 & -3.4035241 & -5.884661 \\
\hline 0.811278 & 0.508452 & 0.907490319 & 73.6412 & 49.68379537 & 66.47034115 & 0.1046350 & -0.1704641 & -4.3457809 & -5.826777 \\
\hline 0.857148 & 0.221947 & 0.84110252 & 16.33194 & 21.2440145 & 62.73791885 & 0.1000946 & 0.3474128 & -0.5169792 & -3.470030 \\
\hline 0.857148 & 0.326574 & 0.879105218 & 43.75766 & 32.5032206 & 66.44422918 & 0.1890595 & 0.0713095 & -4.2289869 & -4.594839 \\
\hline 0.877437 & 0.223242 & 0.842320883 & 15.0707 & 22.77340865 & 62.24210513 & 0.2186415 & 0.5012439 & -0.2745820 & -2.268440 \\
\hline 0.896038 & 0.251519 & 0.786288814 & 12.00099 & 22.2122417 & 45.01107895 & 0.4321139 & 0.5511574 & -4.4572852 & -4.154114 \\
\hline 0.912999 & 0.677808 & 0.752756493 & 26.73433 & 37.74078211 & 53.74349052 & 0.130 & -0.1318042 & -5.8658498 & -6.054064 \\
\hline 0.912999 & 0.231399 & 0.76725192 & 7.327755 & 18.24317062 & 56.70292787 & 0.0697272 & 0.4173124 & -1.1452122 & -2.464949 \\
\hline 0.942163169 & 0.3050235 & 0.79962973 & 51.37625 & 48.00165751 & 69.90163404 & 0.0199069 & 0.3600984 & -6.1528078 & -3.918190 \\
\hline
\end{tabular}

